05

\title{
Ограничение допустимой коэрцитивной силы, вызванное полем рассеяния магнита
}

\author{
() Н.С. Моисеева, ${ }^{1}$ И.И. Резчикова, ${ }^{1,9}$ Д.В. Королев, ${ }^{1}$ Р.Б. Моргунов, ${ }^{2}$ В.П. Пискорский ${ }^{1}$ \\ ${ }^{1}$ Всероссийский институт авиационных материалов, \\ 105005 Москва, Россия \\ ${ }^{2}$ Институт проблем химической физики РАН, \\ 142432 Черноголовка, Россия \\ T e-mail: rezchikovaii@mail.ru
}

Поступило в Редакцию 29 ноября 2018 г.

В окончательной редакции 29 ноября 2018 г.

Принято к публикации 22 января 2019 г.

Показано, что форма образца определяет нижний допустимый предел коэрцитивной силы материала, который может быть использован для изготовления магнита данной формы. На примере колец с радиальной намагниченностью показано, что они могут быть изготовлены только из редкоземельных сплавов, имеющих достаточно высокую коэрцитивную силу для того, чтобы максимально допустимая температура эксплуатации магнита удовлетворяла техническим требованиям.

DOI: $10.21883 /$ JTF.2019.07.47799.413-18

\section{Введение}

В навигационной технике используют постоянные магниты для создания поля в рабочем зазоре прибора. Для динамически настраиваемых гироскопов изготавливают кольцевые магниты с радиальной текстурой. Для акселерометров необходимы магниты в виде призм, аксиально намагниченных цилиндров и аксиально намагниченных кольцевых магнитов. Магниты, работающие в открытой магнитной цепи, всегда находятся в размагничивающем поле, создаваемом поверхностными магнитными зарядами на поверхности магнита. Размагничивающее поле зависит от размагничивающего фактора магнита, который, в свою очередь, определяется геометрией магнита. Важно отметить, что максимальная рабочая температура магнита определяется конкуренцией размагничивающего поля и магнитной анизотропии, которая уменьшается с ростом температуры. Поэтому изменение размагничивающего поля, вызванное изменением формы образца, влияет также и на максимальную рабочую температуру магнита. В работе [1] вводится определение максимальной рабочей температуры магнита, как такой температуры, нагрев до которой уменьшает потокосцепление образца на 5\%. Для определения рабочей точки проводят измерение потокосцепления образца $\psi 1$ в катушках Гельмгольца перед нагревом и потокосцепление образца $\psi 2$ после нагрева и охлаждения до комнатной температуры. Вычисляют величину необратимых потерь $L$ после нагрева по формуле

$$
L=((\psi 1-\psi 2) / \psi 1) \cdot 100 \% .
$$

Если после нагрева (и охлаждения до комнатной температуры) величина необратимых потерь достигает 5\%, то эта температура называется максимальной рабочей температурой $\left(T_{w}\right)[1]$. В работе [1] было установлено, что $T_{w}$ зависит от геометрии магнита, поскольку от геометрии зависит величина размагничивающего фактоpa $(N)$.

Под рабочей точкой магнита $(P)$ подразумевается величина [1]:

$$
P=\frac{4 \pi}{N}-1
$$

Откуда находим $N=4 \pi /(P+1)$.

Так, у магнита из материала $\mathrm{Nd}\left(\mathrm{Fe}_{0.80} \mathrm{Co}_{0.12} \mathrm{~B}_{0.08}\right)_{5.5}$ в форме цилиндра и в форме призмы (с величиной $P=2) T_{w}$ для цилиндра составила $60^{\circ} \mathrm{C}$, для призмы $80^{\circ} \mathrm{C}[1]$. Величина $T_{w}$ зависит от температуры Кюри $\left(T_{C}\right)$ и коэрцитивной силы по намагниченности $(\mathrm{Hci})$ [2]. Так, магнит состава $\mathrm{Nd}_{34.5} \mathrm{Dy}_{1.5} \mathrm{Fe}_{61.5} \mathrm{Al}_{1.2} \mathrm{~B}_{1}$ с величиной $H c i$ более $1600 \mathrm{kA} / \mathrm{m}$ имел $L=0.88 \%$ при $150^{\circ} \mathrm{C}$, а магнит состава $\mathrm{Nd}_{35.4} \mathrm{Fe}_{62.3} \mathrm{~B}_{13}$ с $\mathrm{Hci}=704 \mathrm{kA} / \mathrm{m}$ имел $L=59.21 \%$ [2]. Среди современных магнитов высокие значения $T_{w}$ имеют магниты из сплава $\mathrm{Fe}-\mathrm{Cr}-\mathrm{Co}$. Их $T_{C}=850^{\circ} \mathrm{C}, T_{w}=500^{\circ} \mathrm{C} \quad[3]$. Магниты ЮНДК $(\mathrm{Al}-\mathrm{Ni}-\mathrm{Co}-\mathrm{Fe})$ имеют $T_{C}=890^{\circ} \mathrm{C}$ и $T_{w}=550^{\circ} \mathrm{C}[4,5]$. Магниты $\mathrm{Sm}_{2} \mathrm{Co}_{17}$ имеют $T_{C}=800^{\circ} \mathrm{C}$ и $T_{w}=300^{\circ} \mathrm{C}$ [6]. Магниты $\mathrm{SmCo}_{5}$ имеют $T_{C}=725^{\circ} \mathrm{C}$ и $T_{w}=250^{\circ} \mathrm{C}$ [7]. Таким образом, величина максимальной рабочей температуры магнита определяется его температурой Кюри, коэрцитивной силой по намагниченности и величиной его рабочей точки. Следовательно, для магнитов заданного состава и с известными магнитными характеристиками эксплуатационные свойства зависят только от величины рабочей точки. Следует также отметить, что во всех магнитах за исключением магнитов эллипсоидальной формы величина размагничивающего поля $\left(H_{D}\right)$ неоднородно распределена внутри магнита [8]. Для магнитов разной геометрии величина Р может быть вычислена по методике, предложенной в работе [9]. 

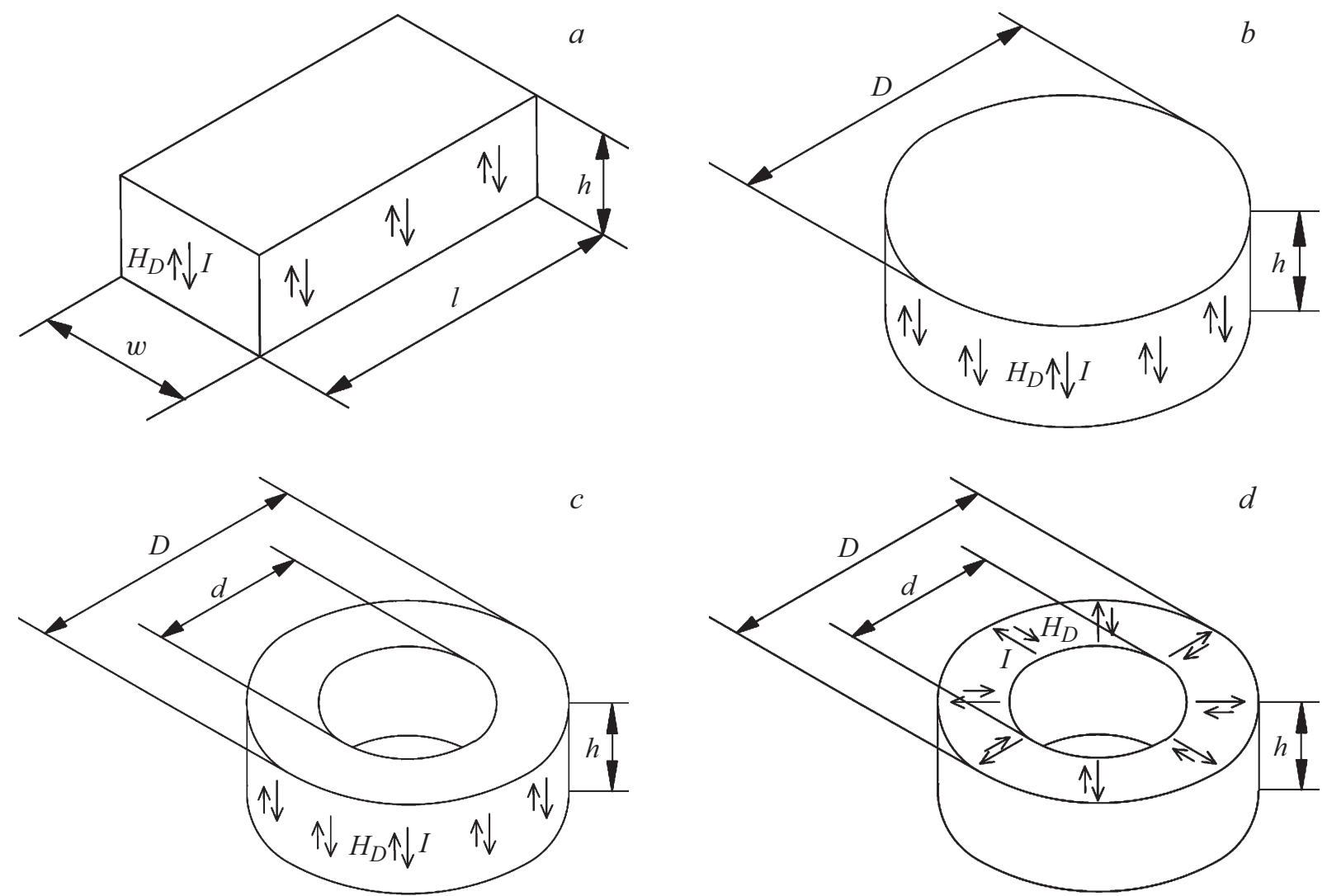

Рис. 1. Геометрия магнитов: $a-$ призматический магнит, $b-$ цилиндрический магнит с аксиальной текстурой, $c-$ кольцевой магнит с аксиальной текстурой, $d-$ кольцевой магнит с радиальной текстурой.

В работе [9] показано, что величина рабочей точки может быть записана следующим образом:

$$
P=\frac{T}{A} \times \sqrt{\frac{\pi}{2} S}
$$

Здесь $T$ - размер магнита в направлении намагничивания или текстуры, $A$ - площадь среднего сечения магнита перпендикулярно текстуре, $S$ - общая площадь поверхности магнита. Таким образом, не любые материалы могут быть использованы для создания сложных по форме магнитов, так как поле рассеивания может сделать тот или иной материал в принципе непригодным. Настоящая работа посвящена вычислению рабочей точки магнитов различной геометрии и определению тех типов магнитных сплавов, которые пригодны для создания кольцевых магнитов, используемых в прецизионной технике.

Настоящая работа выполнена в рамках реализации комплексного научного направления 11.1: „Термостабильные магнитотвердые материалы и математические модели расчета их температурных характеристик для навигационных приборов нового поколения“ („Стратегические направления развития материалов и технологий их переработки на период до 2030 года“) [10].

\section{Результаты исследований}

На рис. 1 представлены типы магнитов. Приведем результаты вычислений рабочей точки с помощью формулы (3) для нескольких типичных геометрий магнитов:

1. Рабочая точка магнита в форме прямоугольной призмы (рис. 1,a) P будет даваться формулой

$$
P=1.77(h / l w)[h(l+w)+l w] 1 / 2,
$$

где $h$ - размер призмы в направлении намагничивания или текстуры, $l$ - длина магнита, $w$ - ширина магнита.

2. Для магнита в форме аксиально намагниченного цилиндра (рис. $1, b)$

$$
P=(2 h / D)[1+(2 h / D)]^{1 / 2},
$$

где $h$ - высота цилиндра, $D$ - его диаметр.

3. Магнит в форме аксиально намагниченного кольца (рис. 1,c)

$$
P=\left[1.596 h /\left(D^{2}-d^{2}\right)\right]\left[(D+d)(D-d+2 h]^{1 / 2},\right.
$$

где $h$ - высота кольца в направлении текстуры, $D-$ внешний диаметр, $d-$ внутренний диаметр.

4. Магнит в форме радиально намагниченного кольца (рис. $1, d)$ имеет рабочую точку

$$
P=[(D-d) / 2 h(D+d)]\left[D^{2}-d^{2}+2 h(D+d)\right]^{1 / 2} .
$$


Здесь $D-$ внешний диаметр кольцевого магнита с радиальной текстурой, $d-$ его внутренний диаметр, a $h$ - высота кольцевого магнита.

\section{Обсуждение результатов исследований}

Зная величину рабочей точки, можно определить из каких магнитотвердых материалов они могут быть изготовлены. Рассмотрим этот вопрос на примере кольцевых магнитов с радиальной намагниченностью. Типичный размер кольцевых магнитов для динамическинастраиваемых гироскопов составляет $D=16.5 \mathrm{~mm}$, $d=12.2 \mathrm{~mm}, h=3 \mathrm{~mm}$. По формуле (7) находим величину рабочей точки $P=0.43$.

Рабочей точке соответствует величина размагничивающего поля $\left(H_{D}\right)$ :

$$
H_{D}=\frac{4 \pi I_{w p}}{1+\frac{B}{H}}
$$

Здесь $4 \pi I_{w p}$ намагниченность в рабочей точке кольцевого магнита с радиальной текстурой (КМРТ). Пусть $4 \pi I_{w p}=0.9 \mathrm{~T}$, тогда $H_{D}=503 \mathrm{kA} / \mathrm{m}$. Отсюда следует, что КМРТ могут быть изготовлены только из редкоземельных магнитов [11-12]. Это связано с тем, что у магнитов $\mathrm{Fe}-\mathrm{Cr}-\mathrm{Co}$ величина $\mathrm{Hci}$ не превышает $88 \mathrm{kA} / \mathrm{m}$ [3]. У магнитов ЮНДК величина $H c i=110-135 \mathrm{kA} / \mathrm{m}$ [13]. У магнитов из материалов системы $\mathrm{P} 3 \mathrm{M}-\mathrm{Fe}-\mathrm{Co}-\mathrm{B}$ (Р3М - редкоземельный металл) величина $\mathrm{Hci}$ составляет более $800 \mathrm{kA} / \mathrm{m}$ [14].

На рис. 2 показана зависимость размагничивающего поля кольцевого магнита с радиальной текстурой от толщины стенки кольца при фиксированных значениях внутреннего диаметра и высоты: $d=12.2 \mathrm{~mm}, h=3 \mathrm{~mm}$. Из рисунка видно, что размер кольцевого магнита из материала $\mathrm{P} 3 \mathrm{M}-\mathrm{Fe}-\mathrm{Co}-\mathrm{B}$ не ограничен. Из материалов на основе ЮНДК можно изготавливать кольцевые магниты с радиальной текстурой с толщиной стенки более $16 \mathrm{~mm}$, из материалов на основе $\mathrm{Fe}-\mathrm{Cr}-\mathrm{Co} \mathrm{c}$

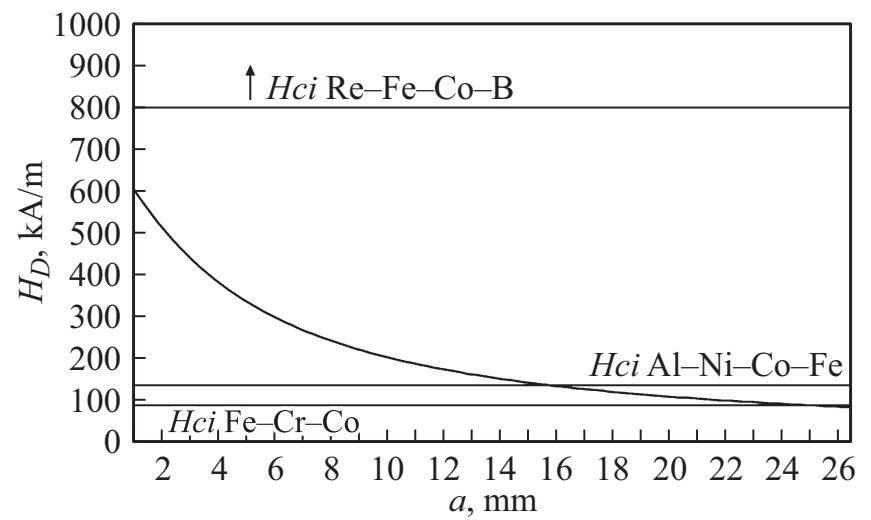

Рис. 2. Зависимость размагничивающего поля кольцевого магнита с радиальной текстурой от толщины стенки кольца при фиксированных значениях внутреннего диаметра и высоты: $d=12.2 \mathrm{~mm}, h=3 \mathrm{~mm}$.

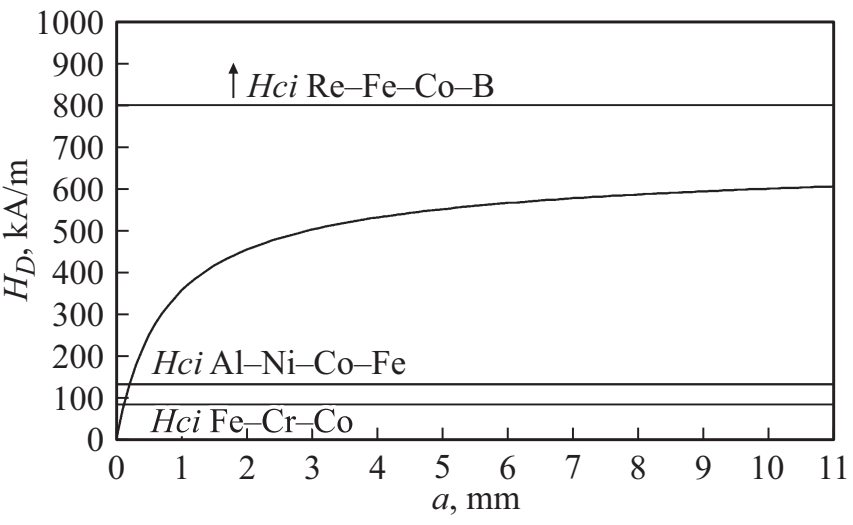

Рис. 3. Зависимость размагничивающего поля кольцевого магнита с радиальной текстурой от высоты кольца при фиксированных значениях внутреннего и внешнего диаметров: $D=16.5 \mathrm{~mm}, d=12.2 \mathrm{~mm}$.

толщиной стенки более $25 \mathrm{~mm}$ при внутренних диаметре и толщине кольца $d=12.2 \mathrm{~mm}, h=3 \mathrm{~mm}$, однако магниты таких размеров в динамически настраиваемых гироскопах не используются.

На рис. 3 показана зависимость размагничивающего поля кольцевого магнита с радиальной текстурой от высоты кольца при фиксированных значениях внутреннего и внешнего диаметров: $D=16.5 \mathrm{~mm}, d=12.2 \mathrm{~mm}$. Из рисунка видно, что размер кольцевого магнита из материала $\mathrm{P} 3 \mathrm{M}-\mathrm{Fe}-\mathrm{Co}-\mathrm{B}$ не ограничен. Из материалов на основе ЮНДК можно изготавливать кольцевые магниты с радиальной текстурой высотой менее $0.2 \mathrm{~mm}$, из материалов на основе $\mathrm{Fe}-\mathrm{Cr}-\mathrm{Co}$ с толщиной стенки высотой менее $0.1 \mathrm{~mm}$ внутреннем и внешнем диаметpax: $D=16.5 \mathrm{~mm}, d=12.2 \mathrm{~mm}$, однако магниты таких размеров в динамически настраиваемых гироскопах не используются и возникают трудности с изготовлением таких магнитов.

Легко показать, используя формулы (4)-(6), что магниты в форме призмы, аксиально намагниченного цилиндра, аксиально намагниченного кольца из материалов $\mathrm{Fe}-\mathrm{Cr}-\mathrm{Co}$ и ЮНДК могут быть изготовлены только с определенным соотношением геометрических размеров, которые и определяют величину размагничивающего поля (см. формулу (8)).

Для типовых размеров магнитов для акселерометров размагничивающее поле представлено ниже.

Для магнитов в виде призм с размерами $h=4 \mathrm{~mm}$, $l=8 \mathrm{~mm}, w=5 \mathrm{~mm}$ величина рабочей точки, согласно формуле (4), составляет $P=1.7$, что соответствует величине размагничивающего поля $H_{D}=267 \mathrm{kA} / \mathrm{m}$ при $4 \pi I_{w p}=0.9 \mathrm{~T}$.

Для магнитов в виде цилиндра с размерами $D=$ $=14.5 \mathrm{~mm}, h=4 \mathrm{~mm}$ величина рабочей точки, согласно формуле (5), составляет $P=0.69$, что соответствует величине размагничивающего поля $H_{D}=426 \mathrm{kA} / \mathrm{m}$ при $4 \pi I_{w p}=0.9 \mathrm{~T}$. 
Для магнитов в виде аксиально намагниченных колец с размерами $D=16.5 \mathrm{~mm}, d=12.2 \mathrm{~mm}, h=3 \mathrm{~mm}$ величина рабочей точки, согласно формуле (6), составляет $P=0.67$, что соответствует величине размагничивающего поля $H_{D}=431 \mathrm{kA} / \mathrm{m}$ при $4 \pi I_{w p}=0.9 \mathrm{~T}$.

\section{Выводы}

1. Получены формулы для вычисления рабочей точки магнитов различной геометрии. Это позволяет устанавливать требования к величине коэрцитивной силы материала магнитов.

2. Показано, что кольцевой магнит с радиальной намагниченностью не может быть изготовлен из материалов $\mathrm{Fe}-\mathrm{Cr}-\mathrm{Co}$ и ЮНДК из-за низкой величины их коэрцитивной силы. Для изготовления магнитов такой формы требуются редкоземельные магниты с высокой коэрцитивной силой.

\section{Список литературы}

[1] Strnat K.J., Xiao Y., Mildrum H.F. // J. Appl. Phys. 1988. Vol. 64. N 10. P. 5522-5524.

[2] Kappel W., Codescu M.M., Popa D. // Roman. Report. Phys. 2004. Vol. 56. N 3. P. 391-398.

[3] Akbar S., Ahmad Z., Awan M.S., Farooque M., Ali A. // Key Engineer. Mater. 2012. Vol. 510-511. P. 507-512.

[4] White E.M.H., Kassen A.G., Dennis K.W., Palasyuk A., Mc Callum R.W., Anderson I.E. // Intern. J. Powder Metall. 2016. Vol. 52. N 4. P. 33-39.

[5] Постоянные магниты. Справочник. Под ред. Ю.М. Пятина. М.: Энергия, 1971. 375 с.

[6] Durst K.D., Kronmuller H., Ervens W. // Phys. Stat. Sol. (A). 1988. Vol. 108. P. 403-416.

[7] Сергеев В.В., Булыгина Т.И. Магнитотвердые материалы. М.: Энергия, 1980. 224 с.

[8] Гуревич А.Г. Магнитный резонанс в ферритах и антиферромагнетиках. М.: Наука, 1973. 588 с.

[9] Parker R.J., Studders R.J. NY., London: John Wiley and Sons, Inc. 1962. $400 \mathrm{p}$.

[10] Каблов Е.Н. // Авиационные материалы и технологии. 2015. № 1. C. 3-33.

DOI: $10.18577 / 2071-9140-2015-0-1-3-33$

[11] Каблов Е.Н., Оспенникова О.Г., Пискорский В.П., Резчикова И.И., Валеев Р.А., Давыдова Е.А. // Авиационные материалы и технологии. 2015. № S2. C. 5-10.

DOI: $10.18577 / 2071-9140-2015-0-S 2-5-10$

[12] Каблов Е.Н., Оспенникова О.Г., Резчикова И.И., Пискорский В.П., Валеев Р.А., Королев Д.В. // Авиационные материалы и технологии. 2015. № S2. C. 24-29. DOI: $10.18577 / 2071-9140-2015-0-S 2-24-29$

[13] Кекало И.Б., Самарин Б.А. Физическое металловедение прецизионных сплавов. М.: Металлургия, 1989. 496 с.

[14] Каблов Е.Н., Оспенникова О.Г., Чередниченко И.В., Резчикова И.И., Валеев Р.А., Пискорский В.П. // Авиационные материалы и технологии. 2015. № S2. С. 11-19. DOI: 10.18577/2071-9140-2015-0-S2-11-19 